air current vary as the square of the relative velocity, the mean reaction is greater than that due to the mean velocity considered as steady.

\section{Herbert Chatley.}

Chinese Government Engineering College,

Tangshan, Noith China, May 24.

\section{Weather Forecasts in England.}

Mr. Mallock, in giving his reasons for believing that correct weather forecasts are not likely to be possible even for twenty-four hours in advance, touches upon many very debatable meteorological theories.

His diagram purporting to show the surface wind currents for an earth, the surface of which is level and uniform, requires for its prediction a much better knowledge of the actual cause of wind distribution than we possess at present. Indeed, at the present time, it would appear that the wind conditions he shows are more nearly those of the northern than the southern hemisphere, and rather are the result of the irregular distribution of land, sea, and mountain than to uniform surface conditions. In the southern hemisphere the conditions, as near the equator, approximate more to belt than to cyclonic conditions.

An inspection of the daily weather charts issued by the Meteorological Office will also show that the general directions of the winds over the northern hemisphere are by no means as Mr. Mallock shows. A cyclone is a much more complex affair than the whirls of his figure. Cyclones are generally rather the result of the common action of several winds moving in different directions. Not only is this the case, but we have no accepted theory as to the cause of cyclones and the source from which they derive their energy.

If it were a simple matter of the passage and rapid appearance and disappearance of cyclones, as $\mathrm{Mr}$. Mallock supposes, I take it that the weather conditions would be rapid alternations of sunshine, cloud, and rain. But such is not the case. We have long periods of fine weather, long periods of wet, unsettled weather, and spells of heat and cold. We must recognise the fact that on the earth we have regions where the weather conditions vary regularly with the seasons, and we also have insular and oceanic weather conditions. The boundaries of these areas are not always the same. The one is apt to encroach upon the other, and it is probable that by obtaining a knowledge of such general movements, weather forecasting for considerable periods of time will be possible.

So long as the old ideas of cyclones and anticyclones held sway, weather predicting really seemed hopeless; but fortunately we find that these old theories, though expressing important truths, require considerable modification in detail. Mr. Mallock's contention that useful forecasting will never be possible seems premature in face of the fact that there are so many things taking place in weather changes the theoretical reasons for which are unknown.

With improved weather charts will come a better knowledge of the theory of cyclones and anticyclones. However, it may never be possible to predict, from the to-be-discovered laws of the winds, the course of weather changes with the certainty the movements of the members of the planetary system can be predicted by the application of Newton's laws.

R. M. DEELEy.

Abbeyfield, Salisbury Avenue, Harpenden.

\section{The Thunderstorm of June 14, at Dulwich.}

My observations on the thunderstorm of Sunday, June I4, at Dulwich, may perhaps be of interest.

Thunder was first heard a little before 12.30 p.m., and lightning was seen from about 12.45. These NO. 2329, voL. 93] continued more or less throughout the afternoon until quite 5 o'clock, the lightning being very brilliant and rather frequent. Heavy rain iell from 12.50 until I.IO, and from I.I5 to about 2.20. Some white hail fell about $r .45$.

At 2 p.m. there was a heavy fall of big hailstones as large as marbles, which lasted about five minutes. Many of these hailstones were like large acid tablets, about an inch long, half an inch broad, and more than a quarter of an inch thick. The hailstones were composed of perfectly clear ice, and did not contain any white opaque substance. Hailstorms are usually accompanied by gusts or squalls of wind; in this storm, however, there was but little wind.

When the big hailstones fell the leaves were torn off the trees, and so the pavements immediately beneath them became quite green with the fallen leaves. The heavy rain, however, quickly washed these away, so that they were carried into the gutters and soon stopped up the drains, with the result that the roads were flooded.

A minute or two after the big hailstones had fallen, a mist arose above the roads and pavements to a height of about $4 \mathrm{ft}$. This clearly showed how the fogs were formed near the Banks of Newfoundland owing to the mixing of the cold and warm sea current.

Rain came on again about 3.15 , and continued until 4 p.m.

It was not able to get to my rain-gauge in Alleyn Park until after 5 o'clock, as the lawn was flooded and the water had not subsided sufficiently for me to get into the garden. I found the rainfall to be $2 \cdot{ }^{2} 5$ in., and of this amount I believe that about $I \cdot 60$ to $I .75$ in. must have fallen in three-quarters of an hour, from I.30 p.m. 10 2.15 p.m.

$$
\begin{gathered}
\text { Royal Meteorological Society, 7o Victoria Street, } \\
\text { London, S.W., June I6. }
\end{gathered}
$$

\section{A Dual Phenomenon with X-Radiation.}

Since our paper on the "X-Rays and Concentration," and our exhibition of models and negatives at the annual general meeting of the Röntgen Society on June 9 , we have obtained further results which favour the hypotheses then suggested.

For instance, if a radiograph be taken at any incidence (except $0^{\circ}$ and $90^{\circ}$ ) of a ring of rectangular cross-section made in ebonite, its circular edges will be distinctly visible as black and white semi-circles. These alternate for the top and bottom outer rings, and they are in the reverse order for the inner circles. These differences were predicted from the generalisa. tion we gave in the paper. We think it would be advantageous to repeat the conclusion of the paper for those who were not present at the exhibition :-

"Generalising our results, it would seem that when $\mathrm{X}$-rays are incident (or emergent) simultaneously upon two surfaces having a common boundary, this will be marked by a white or black band according as the dihedral angle of the solid is greater or less than $180^{\circ}$. If, however, the rays are incident upon one of the surfaces only, and emergent from the other, the order is reversed. In the third case, where the rays are incident upon one surface but parallel to the other (as with single laminæ) the two bands appear in close association, and are observable with difficulty without the aid of suitable magnification." Tangential radiation has given a black or white band according as the surface was convex or concave.

I. G. Rankin.
W. F. D. Chambers.

90 Gordon Road, Ealing. 